\title{
[PHYSICS IN SPACE]
}

\section{GRANULAR MATTER UNDER MICROGRAVITY}

\author{
P. Evesque ${ }^{1}$, Y. Garrabos ${ }^{2}$, A. Garcimartin ${ }^{3}$, N. Vandewalle ${ }^{4}$ and D. Beysens ${ }^{5}$ \\ ${ }^{1}$ CNRS, ECP, Paris, ${ }^{2}$ CNRS, ICMCB, Bordeaux, France, ${ }^{3}$ Univ. Navarra, Spain, ${ }^{4}$ Univ Liege, ${ }^{5}$ CEA-ESPCl, Paris, France
}

T he physics of granular matter under microgravity conditions is important for different fields, ranging from very technological issues such as the management of grains and powders in space, to very fundamental problems like the formation of asteroids or the history of Earth formation. However, in the physics of strongly dissipative systems such as a granular gas, we do not even know if a collection of grains excited by some steady source of energy reaches a steady state or not, and whether the state is unique. Of course, it would be convenient if one could extend the usual thermodynamic concepts of gases and liquids to this kind of matter, then defining under which conditions, with which transfers between the two parts of the system, on which time scale such an extension would be valid.
Here, we consider the problems encountered with granular matter in space and the most realistic way of addressing them by using controlled vibrations to perturb the grain system.

\section{Granular gas excited by vibration}

A question of current interest is: what are the basic properties of granular matter under vibration and weightlessness conditions. The experimental situation is a simple parallelepipedic cell containing a fixed number of grains and the cell walls subjected to harmonic vibrations (frequency $f$, amplitude $A$ ). The grains are then excited by the vibrating cell walls. They can move "randomly" and dissipate energy during grain-grain collisions. 
In micro-gravity

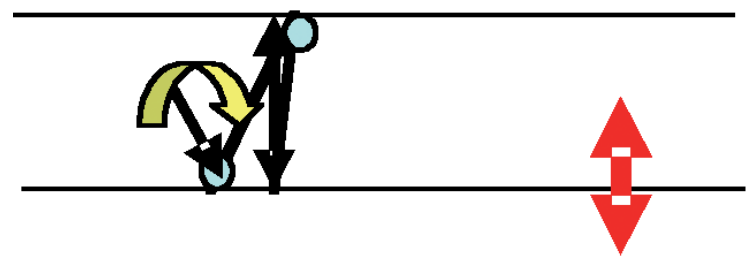

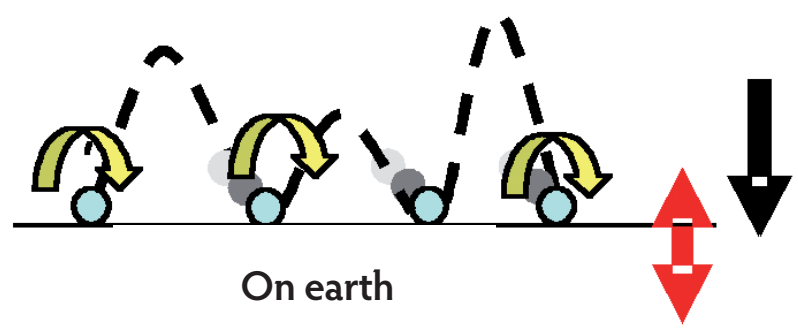

On earth

$\Delta$ FIG. 1: Without gravity (left panel) the rotational energy of the spherical grain is progressively dissipated. Clockwise rotation is induced by the first impact. Upon the second impact, the opposite wall induces a counter clockwise effect. Because of the dissipation of energy on impact, there is only a partial restitution of the rotational energy. The motion eventually becomes 1-D stabilised. On Earth (right panel), if vibrations (red arrows) are applied vertically, gravity (black arrow) is left to play the role of the ceiling wall. Alternating conditions on rotation do not apply and the motion of the grain is not stabilised.

A number of questions then arise: What is the behaviour of each grain? Does a steady state exist for the grain collection? Does the grain system look like a gas, a liquid, a solid? How does it scale with the cell size or the number of particles? Can one observe some kind of gas-liquid or gas-solid transition? What is the distribution of grain velocities? Does the vibrating cell play the role of a thermostat?

We illustrate the subtle complexity of these problems with some examples described in terms of the cell filling ratio. It turns out that when the cell is excited along its longest wall of length $\mathrm{L}$, the main physical parameter is the number $n$ of layers of grains contained in the cell at rest on Earth. Indeed, $L / n \approx l_{c}$ scales as the mean free path $l_{c}$ between grain-grain collisions.

Consider a single spherical grain in a three-dimensional (3-D) cubic box where there is obviously no grain-grain interaction.

In the experiment in weightless conditions, at small vibration amplitude $A$, the grain speed is quite small (and erratic) in the direction perpendicular to vibration, while it is much larger but erratic from one bounce to the next along the vibration direction.

However, we have observed that the motion of the sphere becomes essentially periodic (with some intermittency) once the vibration amplitude exceeds a threshold. This threshold is related to the normal restitution coefficient, that is the ratio of the grain velocities after and before the bounce and is different from one. As the grain moves linearly along a single direction, it makes a round trip within a vibration period, such that its speed is much larger than that of the vibrating cell (i.e. $L f>>\pi A f)$.

In this microgravity experiment, alternate bouncing freezes the grain rotations and motions in the other directions, as illustrated in Figure 1. A finite friction coefficient between particle and wall imposes that a grain with an inclined trajectory rebounds with some rotation on the first surface and with a counter-rotation on the second surface; this process enhances considerably the energy losses and finally freezes the rotational degrees of freedom. A comparison with bouncing on Earth is illustrated on the right panel of Figure 1.

\section{Single grain problem: A simple case?}

A grain moving in a 3-D vibrating box, with two parallel walls oriented perpendicular to the periodic vibrations, is controlled by 13 independent degrees of freedom: 1-time (for vibration), 3 -position- and 3-rotation- coordinates plus 3-translation and 3-rotation speed coordinates. So this is a very complex system similar to the dissipative billiard, except that, when the periodic dynamics exceeds some threshold, it becomes a simple system whose main average position and speed can be predicted.

Of course, the dynamics becomes more complicated if the grain is not perfectly spherical, if the walls are not perfectly flat, or if opposite walls form a tilted-angle. In addition, we understand that when the grain speed $v_{g}$ is large compared to $2 \pi A f$ (or when the restitution coefficient approaches one in numerical simulations), it can randomly collide with the moving wall when the wall goes back or forth. Then, at each bounce, the grain can decrease or increase randomly its speed and the periodic solution looses stability.

\section{Increasing the complexity}

If one performs the experiment with two grains of the same size, both move at the same speed when the motion is periodic (see Fig. 2). When the number of grains is further increased, they start interacting strongly, repelling each other by collision. Their motion then becomes erratic.

The grain dynamics then varies with the number of particle layers $n$ :

- If the number of grains is small (i.e., $n<1.5$ layers), the trajectories look ergodic, which means that on average, the motions of the grains all look similar with the same statistical distribution in space and speed, but there is no long-term correlation.

V FIG. 2: Left panel: Snapshot of two grains moving at the same speed in a vibrated cell under weightness conditions (the direction of vibration is indicated by the white double arrow). Both particles collide only with the walls and make a round-trip per vibration period. Their speed is then much larger than the wall speed and their motion remains "coherent", that is, in phase with the vibration. Right panel: Many grains that collide with the walls and with each other. The grain density is higher in the middle of the cell, and correspondingly the local temperature is lower (vibration amplitude $A=0.3$ $\mathrm{mm}$ and frequency $\mathrm{f}=60 \mathrm{~Hz}$. Experiments on Maxus [5] sounding rockets).
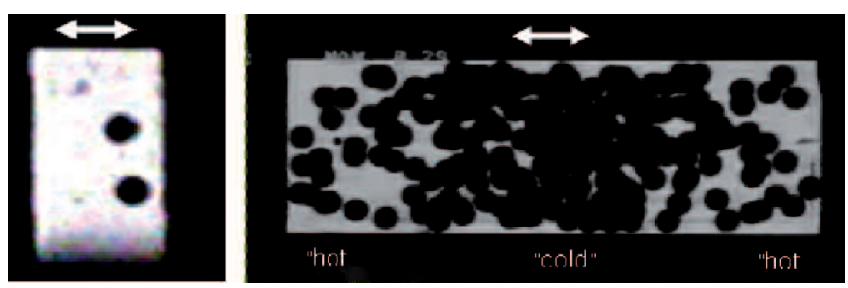


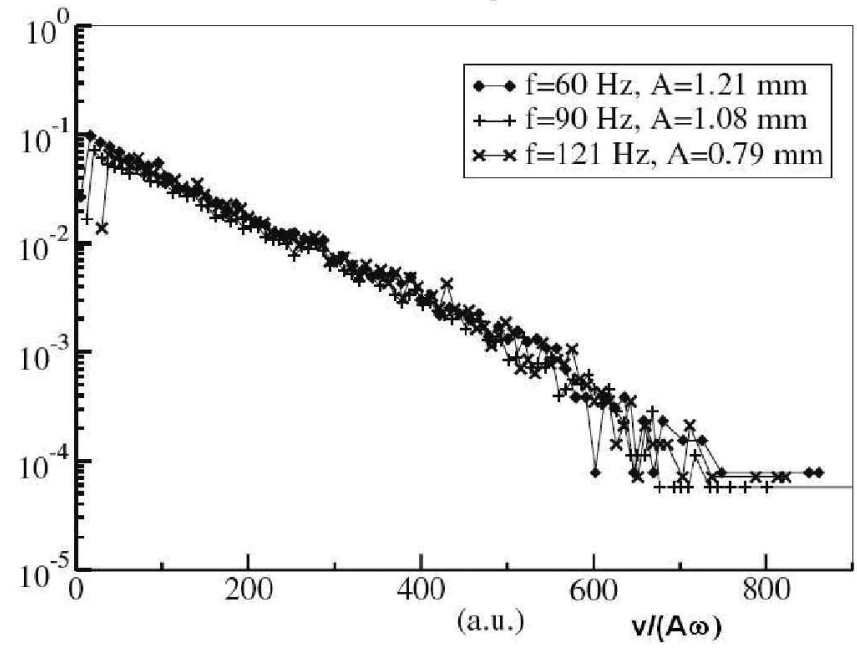

$\Delta$ FIG. 3: The probability distribution function $p(v)$ of impacts at speed $v$ on a fixed plane was measured in a cell containing from 0.5 to 1.5 layers of spherical grains excited by the sinusoidal motion of a piston, with variable frequency $f$ and amplitude $A$. This distribution $p(v)$ follows an exponential law $\exp \left(-v / v_{0}\right)$ with $v_{\circ}$ varying linearly as a function of the maximum boundary speed $A \omega=2 \pi A f$. As the distribution $f(v)$ of speed in the cell is related to $p(v)$ by $p(v)=v f(v), f(v)$ varies as $(1 / v) \exp \left(-v / v_{0}\right)$. As a result, the number of grains with low speed is larger than the equilibrium Boltzmann distribution in a gas $f(v)=\exp \left(-v^{2} / v^{2}{ }_{o}\right)$. The data shown are for a 48-grains case, i.e. 1.5 layers. The exponential decay is found to vary with the number of grains $\mathrm{N}$ : the typical impact speed $\langle v\rangle$ decreases as $\mathrm{N}$ is increased; it's value $\langle v\rangle$ is $\langle v\rangle \approx A \omega$ for $N=0.5$ layer.

This low number of particles fills uniformly most of the available space, except for the zones covered by the moving walls. However, this system is not completely random and the speed distribution is different in 3-D and 2-D cells. We also found (see Fig. 3) that fast grains exhibit a speed distribution $f(v)=$ $(1 / v) \exp \left(-v / v_{o}\right)$, which is different from the Boltzmann-Gibbs equilibrium distribution $f_{B}(v)=\exp \left(-v^{2} / v_{0}^{2}\right)$. This result should not be too surprising since an excited granular system is a nonequilibrium system. However, in most theoretical approaches the equilibrium approximation was used before these results were presented. The non-Boltzmanian distribution should be due to the significant effect of the grain-wall collisions which increase the number of grains with low and large velocities as compared with classical gas particle velocity distribution.

- For systems with larger numbers of grains corresponding to between 1.5 to 3 layers, an in-homogeneous gas is observed. Grains do not have the same kinetic energy (temperature) everywhere; they are slower in the middle of the cell and faster at the boundaries (where the kinetic temperature is higher). In this range of concentration, the particles always have a speed smaller than the boundary velocity. In a granular gas, we note that the information propagates from grain to grain through grain-grain collisions. Hence the speed of sound is typically related to the mean speed of grains. As it is lower than the speed of the walls, the excitation then becomes "supersonic" (but so far, neither compression nor shock waves have been detected).

Many new insights were gained from these experiments, especially the main role of energy losses due to the rotation of the grains. As firstly pointed out in the unexpected 1-ball coherent behaviour, freezing rotation selects and stabilises the linear trajectory of the ball. But when the number of grains is large, rotation effects are dominant in grain-grain collisions. Since these are not frontal collisions, some degrees of freedom get frozen during collisions and energy losses increase. However, how this increase of energy loss can be taken into account in modelling and numerical simulations remains unknown. For example, the speed distribution presented in figure 3 , which was measured at low grain density, could not be reproduced by simulations using the normal restitution coefficient.

At rather high grain density, dynamic clustering or phase separation can be observed in such simulations, often starting with a uniform cloud and a given Boltzmann speed distribution. The present investigations show that such initial conditions are rather unrealistic, or at least difficult to create experimentally. These results trigger quite a number of questions. Should a "mean" restitution coefficient or some other parameters and mechanisms be considered? Can the $f(v)=(1 / v) \exp \left(-v / v_{o}\right)$ distribution be considered as a precursor of the clustering or the intermixed phases? How does it lead to phase separation, if it occurs, when the number of grains is increased?

\section{The need for long duration experiments with high quality microgravity}

A large spectrum of experiments should be undertaken to explore the whole parameters space. These include in particular the vibration parameters $(A, f)$, the number, the size and the material of grains and the cell dimensions. High quality weightlessness is required as soon as the number of grain layers exceeds 2 or 3, otherwise g-jitters can prevent a steady-state to be reached or maintained (as was already observed during parabolic flights). For the immediate future, a number of problems have been selected. These are concerned with the dynamical behaviour in two-dimensional samples, the onset of convection in dense samples, the segregation process in two-species materials, the characterisation of the impact of grains on a plate and of a large number of balls on a packed granular system. These experiments would give at least some clues to some of the important problems encountered in the study of granular materials.

\section{References}

[1] P. Evesque et al. "Current trends on the mechanics of granular matter excited by vibrations in microgravity", Submitted to Microgravity Sc. Technol. (Oct. 2007)

[2] P. Evesque et al., Microgravity sci. technol. XVI-1, 280-284 (2005)

[3] M. Leconte et al., Journal of Statistical Mechanics: Theory and experiment, P07012 (2006)

[4] J. Eggers, Phys. Rev. Lett. 83, 5322-25, (1999)

[5] J. Maddox, Nature 247, 903, (2002)

[6] I. Goldhirsh \& G. Zanetti, Phys. Rev. Lett. 70, 1619-22, (1993)

[7] A. Garcimartin, D. Maza, J.L. Ilquimiche \& I. Zuriguel, Phys. Rev. E 65, $031303(2002)$

[8] G. Lumay \& N.Vandewalle, Phys. Rev. Lett. 95, 028002 (2005) 